UDK 821.131.1-94.09Baretti G.

\title{
RACCONTARE LA CRITICA. \\ L'INTERPRETAZIONE DI MODE E MODI LETTERARI EUROPEI DEL SETTECENTO NELLA FRUSTA LETTERARIA DI GIUSEPPE BARETTI.
}

\author{
Ursula Reuter-Mayring
}

\begin{abstract}
Giuseppe Baretti's literary journal, La Frusta letteraria (1763-65), is a poetic piece of fiction about the contemporary world of literature in the 2nd part of the Eighteenth century. Baretti performs in it his criticism on contemporary literature as well as on the conventional and common processes implicit in literary scene at that time, reflecting also on his own experiences and observations as a 'modern' and cosmopolitan author. To stage this he uses various literary models which were traditional or 'in vogue' at that time in Europe. A critical analysis also reveals the journal in whole to be a closed text constructed strictly according to literary principles, which establishes it as a cohesive piece of poetic art.
\end{abstract}

Key words: Baretti, La Frusta letteraria, journal

Giuseppe Baretti ${ }^{1}$ fu senz'altro uno dei veri cosmopoliti settecenteschi. Per diversi decenni il piemontese visse fuori del paese nativo e viaggiò in diverse parti dell'Europa come tanti altri artisti, scrittori e letterati del suo tempo. Durante tutta la sua vita ebbe un occhio acuto per i fenomeni culturali e sociali dell'ambiente in cui si trovava insieme ad una mente sveglia per recepire quei fenomeni e un'attitudine individualistica e forse altera. Di questa vena sono impregnate fortemente le sue opere letterarie. Per illustrare come esistessero degli "spazi letterari in contatto" non nel senso strettamente geografico ma nella ricezione ed elaborazione letteraria di questo letterato (che, come tanti in

\footnotetext{
${ }^{1}$ Giuseppe Baretti (1719-1789), autore di critica letteraria (a parte la Frusta letteraria è suo per esempio anche il Discours sur Shakespeare et sur monsieur de Voltaire, 1777) e di un'edizione di Machiavelli (1772), dell'epistolario Lettere familiari ... (1762-63), di letteratura odeporica, come An Account of the Manners and Customs of Italy ... (1768) o A Journey from London to Genoa, through Portugal, Spain and France (1770), di numerose opere di carattere pragmatico-linguistico e di diversi dizionari, di traduzioni, per esempio di brani di Orazio, Cervantes e Corneille, e scrittore di poesia, per esempio de Le piacevoli Poesie (1750), parzialmente nella tradizione bernesca. La sua intera opera include ca. 37 libri, più le loro traduzioni contemporanee in diverse lingue europee, pubblicate già in vita. Scrisse in italiano, inglese, francese e spagnolo.
} 
quel periodo, fu un intellettuale 'europeo'), presenterò alcune osservazioni sul contesto di mode e modi letterari europei e come questi si rispecchino nel piano poetico della rivista La Frusta letteraria di Baretti ${ }^{2}$ (cap. 3.). Sono precedute da alcune annotazioni generali su Baretti e la Frusta letteraria (cap. 1.) e da un abbozzo sulla struttura della Frusta letteraria (cap. 2.).

\section{ANNOTAZIONI GENERALI SU BARETTI E LA FRUSTA LETTERARIA}

Giuseppe Baretti fu un rinnovatore della critica letteraria - come dice Walter Binni (Binni 1969, 45) - e secondo Bruno Anglani è l'autore "di alcuni dei libri più belli del Settecento italiano" (Anglani 1997, 11). La sua rivista La Frusta letteraria si era guadagnata una certa fama in Europa quando egli era ancora in vita; veniva citata non solamente da Alfieri e più tardi da Foscolo, ma anche da Lessing (Ritter-Santini 1993, 121). All'inizio del Novecento il nome di Giuseppe Baretti, scelto come titolo della rivista di Piero Gobetti, servì da metafora per una critica letteraria nuova, polemica, battaglie$\mathrm{ra}^{3}$. Nel 2000 nacque infine una rivista online, La Frusta, che dichiara "la ripresa della rivista settecentesca implicita nella [nostra] impresa". La Frusta letteraria di Giuseppe Baretti uscì dall'ottobre del 1763 fino al gennaio del 1765; era stampata e pubblicata a Venezia ("Rovereto") da Antonio Zatta Editore/Stampatore. Come tiratura iniziale - così si afferma - ebbe 6000 esemplari, numero straordinario paragonato alla tiratura di 500 esemplari de Il Caffè (Francioni 1998, LXXXII) o a quella di 1500 esemplari progettati 1793/94 da Goethe e Schiller per Die Horen (Safranski 2009, 100). Il 20 gennaio 1765 fu vietata dai Riformatori dello Studio di Padova a causa di una polemica contro Bembo in cui Baretti l'aveva chiamato "uno de' più magri poeti d'Italia". Successivamente la Frusta letteraria uscì per altri nove mesi - dal febbraio fino al novembre del 1765 - ad Ancona ("Trento") presso Bellelli Editore/Stampatore.

Baretti nacque a Torino nel 1719 e morì a Londra nel 1789. Più di trent'anni della sua vita visse in Inghilterra, la maggior parte del tempo a Londra, in quei tempi la capitale dell'Europa moderna, specialmente in relazione alle idee politiche e letterarie. L'amicizia con Samuel Johnson, con cui condivise la passione per la critica e la filologia, e con David Garrick, il famoso attore di Shakespeare, a cui dovette una ricezione profonda e vivace delle opere shakespeariane, ha lasciato delle impronte ben visibili nella poetica e nelle imprese letterarie di Baretti. Impressionato positivamente dalle condizioni socio-culturali inglesi, Baretti, una volta tornato in Italia, cercò di realizzare a Venezia l'ideale di un autore moderno che scriveva in modo indipendente per un pubblico ed un mercato nuovo - ruolo finora esistente solamente in Inghilterra e in nessun altra società e cultura europea. Durante i suoi diversi soggiorni a Venezia, per tradizione centro prestigioso della stampa e dell'editoria ed un ambiente quasi liberale e tollerante, conobbe i Gozzi ${ }^{4}$ e diventò ammiratore specialmente delle opere teatrali

\footnotetext{
${ }^{2}$ Non saranno però analizzati - in questa relazione - riguardo ai loro significati narrativi e ai valori metaforici o allegorici per La Frusta.

${ }^{3}$ Il Baretti uscì dal 1924 al 1928 a Torino.

${ }^{4}$ Dell' amicizia con Gasparo e Carlo ed anche con la loro sorella Angela, di cui Baretti - a quanto dice s'innamorò e che avrebbe voluto sposare, danno prova gli epistolari sia dei Gozzi sia di Baretti.
} 
di Carlo che vedeva inserirsi anche nella tradizione del grande modello inglese: "il più sorprendente genio che dopo Shakespeare sia comparso in alcun secolo o paese"s.

La rivista di Giuseppe Baretti deve essere considerata come una finzione letteraria. Anche se sembra presentarsi al lettore come strumento di espressione di tante opinioni, in realtà ha un autore unico, Baretti, il cui programma è: raccontare la critica. E l'autore Baretti si descrive come protagonista nell'ambito letterario del Settecento. Concordo con la tesi di Martino Capucci il quale individua la finzione barettiana come l'invenzione dell'autore del giornale e ci nota una "interna coerenza tra l'immaginario autore e ciò che nel giornale ci dice" (Capucci 1998, 748). Ricordiamo che all'epoca nasce il paradigmatico genere moderno: il romanzo. Il pubblico desidera trovare se stesso e le sue condizioni attuali raccontati in una prosa moderna, adeguata e 'poetica'. E viene raccontato tutto: la propria vita e i viaggi fatti sia come grand tour, sia per motivi professionali o politici. La letteratura autobiografico-memorialistica e quella odeporica sono presenti nelle opere di Alfieri, Verri, Goldoni, Gozzi, Casanova ecc. Ma anche la saggistica, perfino quella di carattere scientifico, e le poetiche - o le estetiche, per dirla con un termine nato nel Settecento - vengono scritte seguendo i metodi narrativi del raccontare, sia quelli tradizionali che quelli moderni. Un bell'esempio sono le Lettere virgiliane (1758) di Saverio Bettinelli. Nell'atmosfera illuministica questo raccontare assume la funzione di una messinscena dei contenuti considerati utili della "ansiosa cultura settecentesca del sapere" con il suo "nuovo entusiasmo per le scienze piacevoli" (Stafford 1998, 53 [trad. U. R.-M.]).

\section{LA COSTRUZIONE DELLA FRUSTA LETTERARIA}

La Frusta - intesa come testo unico - può essere analizzata nella sua interezza cercandone i principi narrativi. Troviamo una costruzione formale che rivela due parti principali, ognuna con una struttura schematica fatta di elementi testuali rigidi. Per la prima parte (No. I-XXV) Baretti inventa un racconto di cornice il cui protagonista principale è l'editore immaginario Aristarco Scannabue. In forma di rivista il personaggio di Aristarco presenta le sue critiche, gli articoli di corrispondenti finti, le lettere all'editore e le sue risposte - finte anche queste - così che il lettore trova tutti i tipi testuali caratteristici delle riviste dell'epoca. Sparse fra le parti 'ufficiali', nelle così dette Chiacchiere domestiche, Baretti fa raccontare al suo personaggio Aristarco le storie della sua vita 'privata' in provincia insieme all'amico Don Petronio. Sul palcoscenico della cornice vediamo e sentiamo il processo di ricezione letteraria in una poli-prospettiva: e questa ricezione viene messa in scena proprio come si effettua nei testi e nelle voci della rivista. Allo stesso tempo viene creato uno spazio di periferia tramite la vita straordinaria, quasi esotica di Aristarco, ora 'ritiratosi', che - secondo me - rappresenta una bella soluzione poetica se viene letta come allegoria in nuce della posizione di alterità del critico moderno, solitario e soggettivo, come è stato proposto generalmente dalla critica e approfondito molto bene nel caso di Baretti da Ilaria Crotti (Crotti 1992, 14)

${ }^{5}$ Così nella versione italiana di An Account of the Manners and Customs of Italy ... (1768), si veda Soldini 2004, 98n. 
Nella seconda parte (No. XXVI-XXXIII) Baretti abbandona gli espedienti della cornice e del racconto insieme a tutti gli elementi da rivista: da quel momento in poi pubblica invece i Discorsi. Questi Discorsi fanno parte di una polemica reale fra due autori reali: Giuseppe Baretti e Appiano Buonafede, l'autore del Bue pedagogo, quel libro di critica distruttiva della Frusta pubblicato nel 1764 sotto lo pseudonimo di Luciano da Firenzuola. Per i Discorsi Baretti sceglie un genere ed una voce. In opposizione al carattere aperto e vivace del racconto della prima parte, creato tramite una polifonia di testi e voci, nei Discorsi adopera strategie ben diverse per formare però una eterogeneità simile lavorando con trasgressioni formali alle norme del genere: tradizionalmente il discorso è collegato al contesto erudito e conseguentemente riservato alle spiegazioni serie, alle deduzioni logiche e alle riflessioni teoriche. Baretti invece parla con tanta ironia e vi infila numerosissime divagazioni su aspetti marginali, frantumando così argomenti e frasi. Le strategie narrative del racconto sembrano rispecchiate sul livello testuale del discorso con tutto il suo contesto semantico. La strategia del rispecchiamento può essere individuata sia per la costruzione intera sia per tutti i suoi sub-livelli. E per darne almeno un esempio: le chiacchiere domestiche fra i protagonisti del racconto vengono riprese come dialogo nei Discorsi. Da questa lettura risulta che le due parti stanno in una relazione di testo e metatesto: Il testo funziona come 'racconto di una rivista letteraria' operando con gli elementi narrativi, il metatesto funziona come 'commento' al 'racconto' della prima parte, operando da parte sua con le caratteristiche formali del genere prescelto. La funzione della strategia di rispecchiamento adoperata nell'intera Frusta è quella di stabilire e dimostrare continuamente la coerenza fra testo e metatesto.

\section{IL CONTESTO DI MODE E MODI LETTERARI EUROPEI}

Per realizzare il suo progetto Baretti sceglie poi dei motivi fra i più discussi nell'ambito culturale europeo della sua epoca e applica delle strategie letterarie di gran successo presso il pubblico contemporaneo. Fra le grandi tematiche rintracciabili nella Frusta, rilevabili specialmente il relazione al personaggio di Aristarco, si trovano menzionate per esempio delle riflessioni da opere di Montesquieu e di Rousseau intorno alla relazione fra natura e civilizzazione, insieme al vasto interesse per i luoghi esotici e primitivi, dove si sviluppa l'ideale di un atteggiamento naturale che sta in contrasto con le artificiali norme sociali del mondo europeo: Aristarco ci viene presentato come un grande viaggiatore, che visitò sopratutto l'Oriente e che conobbe bene le culture ed i costumi esotici sia come soldato sia come amico di personaggi di alto rango e come esperto e traduttore di lingue esotiche. Nel momento però in cui noi lettori lo conosciamo quale editore della Frusta ci appare come ritirato dal mondo e vive una vita semplice in provincia, a qualche distanza dalle metropoli. La moda orientale, molto in vigore all'epoca, s'esprime nel suo modo di vestirsi: “[...] s'avvezzò [...] a indossare una lunga zimarraccia [...]" (Baretti I, 246). Ricordiamo che anche il protagonista nella cornice della rivista Il Caffe, Demetrio, proprietario della bottega di caffe, ha addosso la zimarra. Mentre per Demetrio quello è il vestito tradizionale del "levantino", per il

${ }^{6}$ Tutte le citazioni dalla Frusta Letteraria appaiono secondo l'edizione Milano: SA Notari, 1929. 
protagonista barettiano rappresenta invece un segnale di abito diverso - nel doppio significato della parola. E, in conseguenza:

"[...] le donne del villaggio non si curano troppo di trattar familiarmente con Aristarco, e gli uomini anch' essi di rado s'arrischiano a parlargli, tanto più che alcuni lo hanno anche in qualche leggier sospetto di negromante, o, come dicono essi, di stregone [...]." (Baretti, I, 37)

Così come la tematica della diversité, che appare in queste righe, altrettanto erano molto presenti nelle conversazioni dei salotti le riflessioni sulla fisiognomica, che si sarebbero condensate infine nell'opera di grande successo dello svizzero Lavater ${ }^{7}$, e quelle su una nuova relazione fra fisico e intelletto e sulla teoria del clima, specialmente su la chaleur, promosse da Montesquieu ${ }^{8}$ (Richter 2009, 129). Di tutto questo si trovano tracce nella descrizione della 'creazione' di Aristarco:

"La vita di quella mansueta ed innocua gente, che noi volgarmente chiamiamo letterati, non è, e non può essere, gran fatto piena di strani accidenti, né troppo feconda di maravigliose varietà, perché è per lo piú una vita vissuta tutta in un paese solo, e tutta limitata in un ristretto cerchio d'amici, la maggior parte ignoranti affatto o appena iniziati negli elementi del sapere. Ma la vita del nostro Aristarco Scannabue è stata una cosa assai diversa, ve l'assicuro. Quando alla Madre Natura venne in capriccio di formare il suo individuo, parve proprio si proponesse di fare una singolar cosa, poiché gli è certo che si stette di molte settimane rimescolando assai ignee materie, che infuse quindi nella sua corporea sostanza. E quando l'ebbe tutto formato in guisa da farlo poi riuscire, come riuscì di fatto, un uomo di statura poco meno che gigantesca, quella buona Madre Natura lo produsse al mondo in uno de' più ardenti giorni della canicola; onde non è da stupirsi se Aristarco non potette poscia stare per un lungo tempo fisso in un luogo, e se de' quindici lustri già da esso vissuti ne passò dieci intieri intieri sempre avvolgendosi come una fiamma per diverse regioni del mondo." (Baretti, I, 33)

Già la "buona Madre Natura" in sé è motivo interessante perché potrebbe far riferimento al cambiamento di un paradigma socio-filosofico intorno al concetto di natura che piglia forza nel contesto dell'illuminismo ${ }^{9}$. Anche i passatempi preferiti di Aristarco, siano quelli della sua gioventù siano quelli dell'età già avanzata, a parte dei loro significati metaforici, richiamano il contesto storico e socio-culturale: sono degli "istruttivi giochi scientifici" che "erano una pratica per la vita perché rappresentavano quell'arte affascinante dell'esperimento considerata così importante sia nei laboratori sia nella vita" (Stafford 1998, 75 [trad. U. R.-M.]). Così vediamo che il giovane Aristarco

"[...] faceva molte e molte giornate in un giardino di casa, diligentemente cercando scorpioni pe' fessi de' muri, e di sottovia de' vasi di creta e di legno, e schiacciando quegli scorpioni se li trovava piccini, o riponendoli vivi in un fiasco d'olio se s'ab-

\footnotetext{
${ }^{7}$ Johann Caspar Lavater: Physiognomische Fragmente zur Beförderung der Menschenkenntnis und Menschenliebe, 4 Bde., 1775-78.

${ }^{8}$ Inclusa ne L'esprit des loix, 1748.

${ }^{9}$ Suggerito da: Merchant, Carolyn: Death of Nature, New York: 1980. (Il primo ed importante cenno è dovuto a Waltraud Pulz, Università di Monaco/Germania).
} 
battevano ad esser grandi, pigliandoli sempre su colle sue proprie dita, senza punto di paura delle loro velenose code." (Baretti, I, 33)

E vediamo il vecchio come trova un grande interesse nella relazione fra uomo ed animali:

"La sua giornaliera compagnia e divertimento sono alcuni cani d'Irlanda, e del Canadà, alcuni gatti d'Angola e del Malabar, e alcuni uccelli e scimmiotti di varie parti d'America, tutti nati da altri cani, gatti, uccelli e scimmiotti recati con sè quando tornò da quelle regioni. [...] A ognuno di quei scimmiotti [...], egli ha capricciosamente posto un nome di poeta o di prosatore moderno, secondo il carattere ch'egli crede scorgere in questo o in quell'altro scimmiotto [...]." (Baretti, I, 35)

Infatti fu nel primo Settecento che arrivarono in Europa i primi scimpanzé ed orangotanghi vivi (Watson 2005, 860), oggetto di un vivo e diffuso interesse scientifico per esempio da parte di Carl von Linné o di Edward Tyson; quest'ultimo stabilì le somiglianze e le differenze in uomo e scimmia e pubblicò Orang-outang, sive, Homo sylvestris: or, The anatomy of a pygmie compared with that of a monkey, an ape, and a man (1699).

Accenti forti della assai diffusa moda inglese si trovano nel corrispondente Onesto Lovangelo [sic!] e nella "dama inglese intendentissima della nostra lingua" o come viene chiamata anche, la "bella Lady". La corrispondenza dei due personaggi viene pubblicata di Aristarco nella Frusta come esempio della modernità del pubblico letterario inglese - oggetto delle loro lettere è uno dei testi più discussi dell'epoca, la Letter on Enthusiasm di Shaftesbury (1708). Quanto alla settecentesca moda del caffè, ampliamente diffusa in tutta Europa, è già stata profondamente considerata dalla critica riguardo alla sua potenza metaforica.

“[...] quella bottega del Caffè linda e odorosa in cui si incontrano illuminati e uomini qualunque - absit iniuria verbo -, torpidi benpensanti e sensibili progressisti in una lieve trama scenica di ingenua efficacia, con signori sconosciuti che di colpo rivelano le loro brillanti qualità e lasciano a bocca aperta i rappresentanti dei luoghi comuni, con il levantino Demetrio, generoso e impeccabile, che trascina la sua zimarra da Lettres persanes e la sua autorizzata originalità di orientale in un milieu di dormienti ai quali il caffè porta la sua eccitazione di facile simbolo settecentesco di modernità attiva e disinvolta nelle volute del suo profumo di moda esotica [...].” (Binni 1969, 41) $)^{10}$

Baretti però oppone alla rivelazione delle "brillanti qualità" e "l'attiva modernità" una scena di privatezza, una scena che può essere letta come contra-disegno a quella pubblicità sveglia e spiritosa dei caffè, e che potrebbe nello stesso momento evocare già certi momenti in cui nella letteratura romantica le figure oltrepasseranno il confine fra realtà e fantastico, a volte bevendo i liquidi-liquori a volte favellando e spesso al crepuscolo o durante le ore notturne come lo fanno per esempio quegli eroi bizzarroromantici di E. T. A. Hoffmann. Baretti narra così le veglie di Aristarco insieme al suo amico Don Petronio Zamberlucco,

${ }^{10}$ Sull'aspetto dello spazio pubblico del Caffè si veda anche Hoeges, 1978. 
“[...] il quale è curato del luogo dov'egli [Aristarco] dimora. Questo dabben religioso si compiace di passare qualche sera di domenica con Aristarco, fumando seco un paio di pipe, aiutandolo con assai modestia a vuotare qualche fiasco e stendendo con molto grave taciturnità gli orecchi quand'egli ciancia de' suoi viaggi, de' suoi tanti pericoli passati, delle mode e costumanze de' lontani paesi, delle varie favelle e della varia letteratura di varie nazioni." (Baretti, I, 37)

Anche con le sue scelte letterarie Baretti ricorre ai modelli en vogue nel mondo letterario europeo del Settecento: La forma della 'nuova' rivista come tante altre dell'epoca segue il modello dei famosi moral weeklies inglesi come per esempio The Spectator. L'ambito delle riviste inglesi era molto familiare a Baretti specialmente tramite l'amicizia coltivata durante il suo lungo soggiorno a Londra con uno dei suoi protagonisti più famosi, Samuel Johnson ${ }^{11}$, lui stesso editore. Dai moral weeklies vengono adottati i nomi parlanti, esprimenti cioè le caratteristiche del personaggio, ed il modello narrativo della cornice - come lo faranno i Verri per il famoso Caffè fino al Borsieri per il Conciliatore. Interpretare queste scelte poetiche di Baretti in una mono-prospettiva sarebbe però inadeguato. Senz'altro per Baretti è anche un ricorrere al repertorio della grande tradizione novellistica italiana con il suo elemento narrativo quasi costituivo della cornice. ${ }^{12}$ È per esempio anche la scelta di Carlo Gozzi, stimatissimo da Baretti come letterario e come amico's ${ }^{13}$ con cui condivide quella "preferenza [...] per linee laterali e risentite della tradizione letteraria italiana" (Ricorda 2001, 17): Gozzi riprende il genere sempre in cerca di una letteratura moderna e nondimeno radicata nella tradizione e collega le sue novelle ${ }^{14}$ se non con una cornice, con "altri fili unificanti" (Ricorda 2001, 21). Il fatto che la Frusta si rivolga ad un nuovo pubblico è visibile nell'uso di 'moderni' elementi pragmatico-testuali come annotazioni esplicative o traduzioni, elementi che nascono proprio nel momento in cui il pubblico diventava più eterogeneo e meno erudito. Le condizioni di un nuovo mercato letterario si mostrano bene, nella Frusta; nei diversi richiami di carattere economico. Come in un tableau Baretti fa vedere nella sua rivista il discorso letterario - reale ed ideale - dei suoi tempi, discorso del quale saranno menzionati almeno due lati: i suoi protagonisti, ovvero l'autore, il critico, il pubblico, specialmente quello femminile, e i suoi registri stilistici, per esempio la polemica nella cui forma vengono effettuate le controversie letterarie più famose dell'epoca, o la corrispondenza, specchio non solo delle relazioni ma anche delle letture private e soggettive, solitarie o fra amici. Sempre nella costruzione del suo protagonista, Aristarco, Baretti gioca su alcuni elementi tipici letterari che corrispondono perfettamente al gusto del pubblico in Europa, siano essi elementi del romanzo - picaresco, avventuroso e epistolare - o dell'autobiografia, genere in cui si presenta l'individuo come oggetto d'interesse e di cui furono scritti esempi straordi-

${ }^{11}$ The Rambler 1750-1752, The Adventurer 1752-1754, The Idler 1758-1760.

${ }^{12}$ Elemento narrativo che, seguendo René Wellek (Wellek/Warren 1995, 240), rimanda direttamente al romanzo moderno.

${ }^{13}$ I rapporti e la stima cambiano col tempo, definitivamente intorno al 1784, si veda la lettera di Baretti a Francesco Carcano del 12 maggio 1784.

${ }^{14}$ Pubblicate come raccolta intera nell'edizione Colombani, "Ed. Ultima”, 1774, della quale Gozzi manda qualche esemplare a Baretti a Londra non senza la speranza di vederle presentate da lui al pubblico inglese o anche tradotte, si veda la lettera di Gozzi a Baretti del 12 aprile 1777 e di Baretti a Gozzi del 9 maggio, per esempio in: Gozzi 2004. 
nari all'epoca, non solo da Rousseau o Casanova, ma, fra altri, anche da Carlo Gozzi e Goldoni. Certamente notevole è quell'elemento picaresco presente nella figura e nella vita di Aristarco, che va anche oltre il suo significato metaforico e funzionale ${ }^{15}$. Ricordiamo che in seguito al Don Quichote (1605/06) i romanzi della tradizione picaresca ebbero un grande successo e trovarono rinnovamento ancora all'inizio del Settecento specialmente in Francia col Gil Blas e con Le Diable boiteux de Alain-René Lesages (Keilhauer 2002, 25).

Al concetto fondamentale del Settecento, in definitiva, alla critica, Baretti risponde con la proposta di una critica letteraria poetica, cioè con l'intera creazione della sua Frusta letteraria. La soluzione poetica gli offriva la possibilità di giocare sui modelli letterari più ricercati nel mondo europeo contemporaneo e dava alla sua critica quel sapore di attualità e di cosmopolitismo che per lui era - ed è rimasto fino ad oggi - caratteristico di una critica letteraria moderna, indipendentemente dai giudizi letterari espressivi. L'autore moderno, che Baretti cercava di essere, lo ritroviamo nella sua invenzione poetica-letteraria, nell'allegoria di quel vecchio protagonista nuovo, Aristarco, un 'io' isolato, un individuo solo e contraddittorio, vuol dire tutto moderno ${ }^{16}:$ il critico.

Klagenfurt, Austria

\section{FONTI}

Baretti, Giuseppe: La Frusta letteraria. I-IV, prefazione di Massimo Bontempelli, Milano: SA Notari, 1929.

: Epistolario. I-II, (ed.) Luigi Piccioni, Bari: Laterza, 1930.

Gozzi, Carlo: Lettere, (ed.) Fabio Soldini,Venezia: Marsilio, 2004.

\section{STUDI}

Anglani, Bartolo: Il mestiere della metafora. Giuseppe Baretti, intellettuale e scrittore, Modena: Mucchi, 1997.

Binni, Walter: "Giornali letterari del settecento", in: Critici e poeti dal cinquecento al novecento, Firenze: La Nuova Italia, 1969.

Capucci, Martino: "La prosa narrativa, memorialistica e di viaggio. Avventurieri e poligrafi. Letterati, critici, polemisti”, in: Enrico Malato (ed.): Storia della letteratura italiana, Vol. VI: Il Settecento, Roma: Salerno, 1998.

Crotti, Ilaria: Il viaggio e la forma. Giuseppe Baretti e l'orizzonte dei generi letterari. Modena: Mucchi, 1992.

Fido, Franco: 'Don Chisciotte giornalista: La 'Frusta'del Baretti e la vita culturale veneziana a metà del settecento", in: id.: Il paradiso dei buoni compagni. Capitoli di storia letteraria veneta, Padova: Antenore, 1988.

Francioni, Gianni: "Storia editoriale del 'Caffe' ", in: Gianni Francioni; Sergio Romagnoli (ed.): Il Caffé. 1764 - 1766, Torino: Bollati Boringhieri, 1998.

\footnotetext{
${ }^{15} \mathrm{Su}$ questo aspetto si vedano specialmente Crotti 1992, 179 e Fido 1988.

${ }^{16}$ Fenomeno rilevato molto bene da Crotti 1992.
} 
Hoeges, Dirk: Aufklärung und die List der Form. Zur Zeitschrift 'Il Caffé' und zur Strategie italienischer und französischer Aufklärung, Krefeld, 1978 (= Schriften und Verträge des Petrarca Instituts, Köln, XXVIII).

Keilhauer, Annette: Der Lügner, der Wahrheiten erzählt. Alain-René Lesages 'Der hinkende Teufel' als Ausdruck eines neuen dichterischen Selbstverständnisses, in: Richard van Dülmen; Sina Rauschenbach (ed.): Denkwelten um 1700, Köln: Böhlau, 2002.

Piccioni, Luigi: Bibliografia analitica di Giuseppe Baretti, Torino: Società Subalpina, 1942.

Ricorda, Ricciarda: "Introduzione”, in: Gozzi, Carlo: Novelle, (ed.) Ricciarda Ricorda, Venezia: Marsilio, 2001.

Richter, Dieter: Der Süden. Geschichte einer Himmelsrichtung, Berlin: Wagenbach, 2009.

Ritter-Santini, Lea (ed.): Eine Reise der Aufklärung. Lessing in Italien 1775 (= Katalog der Ausstellung vom 21.8. - 17.10.1993 in Wolffenbüttel), Berlin: Akademie Verlag, 1993.

Safranski, Rüdiger: Goethe und Schiller. Geschichte einer Freundschaft, München: Hanser, 2009.

Stafford, Barbara Maria: Kunstvolle Wissenschaft. Aufklärung, Unterhaltung und Niedergang der visuellen Bildung, Amsterdam \& Dresden: Verlag der Kunst, 1998.

Watson, Peter: Ideen, München: Bertelsmann, 2005 (Ideas. A History from Fire to Freud, London: Random House, 2005).

Wellek, René / Warren, Austin: Theorie der Literatur, Weinheim: Beltz Athenäum, 1995 (Theory of Literature, 1949).

\section{WEBLINKS}

http://www.lafrusta.net/ [05.09.2011] 